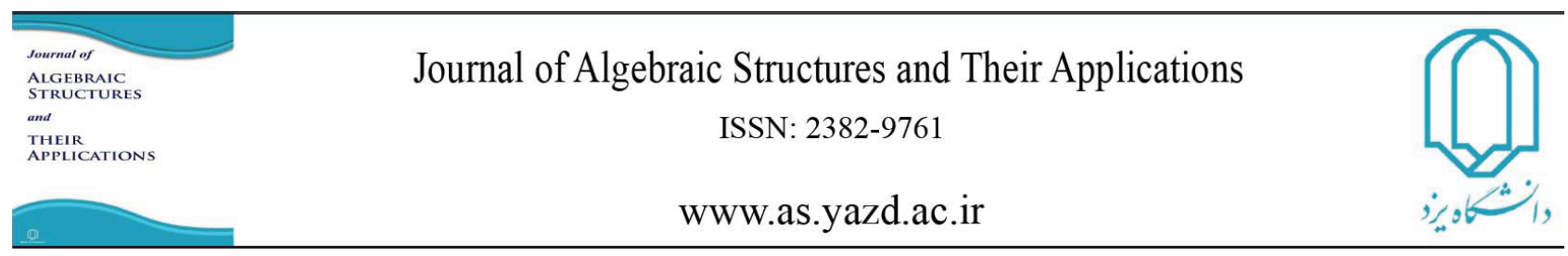

Algebraic Structures and Their Applications Vol. 4 No. 2 ( 2017 ) pp 27-38.

\title{
ON THE EIGENVALUES OF NON-COMMUTING GRAPHS
}

\author{
MODJTABA GHORBANI, ZAHRA GHARAVI-ALKHANSARI AND ALI ZAEEM-BASHI
}

Communicated by S. Alikhani

\begin{abstract}
The non-commuting graph $\Gamma(G)$ of a non-abelian group $G$ with the center $Z(G)$ is a graph with the vertex set $V(\Gamma(G))=G \backslash Z(G)$ and two distinct vertices $x$ and $y$ are adjacent in $\Gamma(G)$ if and only if $x y \neq y x$. The aim of this paper is to compute the spectra of some well-known NC-graphs.
\end{abstract}

\section{INTRODUCTION}

All graphs considered in this paper are simple namely undirected graph without parallel edges. Also, all graphs and groups are finite. Let $G$ be a non-abelian group with the center $Z(G)$. The non-commuting graph $(N C$-graph) $\Gamma(G)$ is a graph with the vertex set $G \backslash Z(G)$ and two distinct vertices $x, y \in G \backslash Z(G)$ are adjacent whenever $x y \neq y x$. The concept of $N C$-graphs was first considered by Paul Erdős in 1975 to answer a question on the size of the cliques of a graph, see [21]. For background materials about $N C$-graphs, we encourage the reader to see references [1, 112, 1.9, [20].

DOI :http://dx.doi.org/10.29252/asta.4.2.27

MSC(2010): 22E46, 53C35, 57S20.

Keywords: non-commuting graph, characteristic polynomial, center of group.

Received: 01 Aug 2017, Accepted: 16 May 2018.

*Corresponding author: mghorbani@sru.ac.ir 
In the next section, we give necessary definitions and some preliminary results and the third section contains the main results on the spectra of $N C$-graphs.

\section{Definitions and Preliminaries}

Our notation is standard and mainly taken from standard books such as [7, $\mathbf{8}, \mathbb{\Pi}]$. For a group $G, \operatorname{Cent}(G)=\left\{C_{G}(x) \mid x \in G\right\}$, where $C_{G}(x)$ is the centralizer of the element $x$ in $G$,

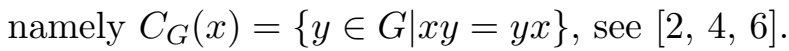

Example 2.1. Consider the symmetric group $\mathbb{S}_{3}$ by the following presentation

$$
\mathbb{S}_{3}=\left\langle a, b: a^{2}=1, b^{3}=1, a^{-1} b a=b^{-1}\right\rangle .
$$

This group is the smallest non-abelian group of order 6 . The center of this group is trivial and so $\mathbb{S}_{3} \backslash Z\left(\mathbb{S}_{3}\right)=\left\{a, b, b^{2}, a b, a b^{2}\right\}$. The element $b$ commutes with $b^{2}$ and thus $\Gamma\left(\mathbb{S}_{3}\right) \cong K_{5} \backslash e$, where $K_{n} \backslash e$ denotes the graph obtained from the complete graph $K_{n}$ by deleting an edge.

An independent set of a graph $\Gamma$ is a subset $S \subseteq V(\Gamma)$ if no two vertices of which are adjacent. The size of the largest independent set is called the independence number. A $k$ partite graph is a graph whose vertices can be partitioned into $k$ different independent sets. When $k=2$ or 3 , the related graph is denoted by bipartite or tripartite graph, respectively.

Let $A=\left[a_{i j}\right]$ and $B=\left[b_{i j}\right]$ be matrices of sizes $m$ by $p$ and $q$ by $n$, respectively. The tensor product (or Kronecker product) of $A$ and $B$ is the $m q$ by pn matrix $A \otimes B$ obtained from $A$ by replacing each entry $a_{i j}$ of $A$ with the $q$ by $n$ matrix

$$
a_{i j} B(1 \leq i \leq m, 1 \leq j \leq p)
$$

The lexicographic product or composition graph $\Gamma_{1}$ o $\Gamma_{2}$ of two graphs $\Gamma_{1}$ and $\Gamma_{2}$, is a graph with the vertex set $V\left(\Gamma_{1}\right) \times V\left(\Gamma_{2}\right)$ and any two vertices $(u, v)$ and $(x, y)$ are adjacent in $\Gamma_{1}$ o $\Gamma_{2}$ if and only if either $u$ is adjacent with $x$ in $\Gamma_{1}$ or $u=x$ and $v$ is adjacent with $y$ in $\Gamma_{2}$. If the adjacency matrices of two graphs $\Gamma_{1}$ and $\Gamma_{2}$ are $A_{m \times m}$ and $B_{n \times n}$ respectively, then the lexicographic product of $\Gamma_{1}$ o $\Gamma_{2}$ has adjacency matrix

$$
A \otimes J_{m}+I_{n} \otimes B
$$

For given graphs $\Gamma_{1}$ and $\Gamma_{2}$ their Cartesian product $\Gamma_{1} \square \Gamma_{2}$ is defined as the graph on the vertex set $V\left(\Gamma_{1}\right) \times V\left(\Gamma_{2}\right)$, where two vertices $u=\left(u_{1}, u_{2}\right)$ and $v=\left(v_{1}, v_{2}\right)$ are adjacent if and only if either $\left(\left[u_{1}=v_{1}\right.\right.$ and $\left.\left.u_{2} v_{2} \in E\left(\Gamma_{2}\right)\right]\right)$ or $\left(\left[u_{2}=v_{2}\right.\right.$ and $\left.\left.u_{1} v_{1} \in E\left(\Gamma_{1}\right)\right]\right)$. Let $A$ and $B$ be square matrices of orders $m$ and $n$, respectively. The adjacency matrix of Cartesian product $\Gamma_{1} \square \Gamma_{2}$ can be written as $A \otimes I_{m}+I_{n} \otimes B$, see [ $[8]$.

The direct product $\Gamma_{1} \otimes \Gamma_{2}$ of two graphs $\Gamma_{1}$ and $\Gamma_{2}$ is defined as the graph on the vertex set $V\left(\Gamma_{1}\right) \times V\left(\Gamma_{2}\right)$ and two vertices $u=\left(u_{1}, u_{2}\right)$ and $v=\left(v_{1}, v_{2}\right)$ are adjacent if and only if 
$u_{1} v_{1} \in E\left(\Gamma_{1}\right)$ and $u_{2} v_{2} \in E\left(\Gamma_{2}\right)$. The adjacency matrix of $\Gamma_{1} \otimes \Gamma_{2}$ is the tensor product $A \otimes B$ of the adjacency matrices of $\Gamma_{1}$ and $\Gamma_{2}$.

Example 2.2. Consider the group $U_{6 n}$ with the following presentation

$$
U_{6 n}=\left\langle a, b: a^{2 n}=1, b^{3}=1, a^{-1} b a=b^{-1}\right\rangle .
$$

The elements of this group are

$$
\left\{1, a, \cdots, a^{2 n-1}, b, b a, \cdots, b a^{2 n-1}, b^{2}, b^{2} a, \cdots, b^{2} a^{2 n-1}\right\} .
$$

One can see that $Z\left(U_{6 n}\right)=\left\langle a^{2}\right\rangle$ and so $\left|Z\left(U_{6 n}\right)\right|=n$. This implies that

$$
\left|V\left(\Gamma\left(U_{6 n}\right)\right)\right|=\left|U_{6 n}\right|-\left|Z\left(U_{6 n}\right)\right|=5 n .
$$

Let $i, j$ be odd numbers, then

$$
\left(a^{i} b\right)\left(a^{j} b\right)=\left(a^{i} b\right) a\left(a^{j-1} b\right)=a^{i}(b a) a^{j-1} b=a^{i+j}=\left(a^{j} b\right)\left(a^{i} b\right) .
$$

Hence, $\left\{a b, a^{3} b, \cdots, a^{2 n-1} b\right\}$ is an independent set. Similarly, we can prove that if $i, j$ are odd numbers, then $\left(a^{i} b^{2}\right)\left(a^{j} b^{2}\right)=\left(a^{j} b^{2}\right)\left(a^{i} b^{2}\right)$ and so the set $\left\{a b^{2}, \cdots, a^{2 n-1} b^{2}\right\}$ is an independent set. Now we can show that the following sets are independent

$$
\begin{aligned}
& \left\{a, a^{3}, \cdots, a^{2 n-1}\right\},\left\{a b, a^{3} b, \cdots, a^{2 n-1} b\right\},\left\{a b^{2}, a^{3} b^{2}, \cdots, a^{2 n-1} b^{2}\right\}, \\
& \left\{b, b^{2}, a^{2} b, a^{2} b^{2} \cdots, a^{2 n-2} b, a^{2 n-2} b^{2}\right\} .
\end{aligned}
$$

This implies that $\Gamma\left(U_{6 n}\right)$ is a 4-partite graph with the following adjacency matrix

$$
\left(\begin{array}{cccc}
0_{n} & J_{n} & J_{n} & J_{n \times 2 n} \\
J_{n} & 0_{n} & J_{n} & J_{n \times 2 n} \\
J_{n} & J_{n} & 0_{n} & J_{n \times 2 n} \\
J_{2 n \times n} & J_{2 n \times n} & J_{2 n \times n} & 0_{2 n}
\end{array}\right)=\left(\begin{array}{ccccc}
0 & 1 & 1 & 1 & 1 \\
1 & 0 & 1 & 1 & 1 \\
1 & 1 & 0 & 1 & 1 \\
1 & 1 & 1 & 0 & 0 \\
1 & 1 & 1 & 0 & 0
\end{array}\right) \otimes J_{n}=B \otimes J_{n}
$$

where $J_{n}$ is the square matrix with all entries one.

Example 2.3. Consider now the $N C$-graph of group $T_{4 n}$ with the following presentation

$$
T_{4 n}=\left\langle a, b: a^{2 n}=1, a^{n}=b^{2}, b^{-1} a b=a^{-1}\right\rangle .
$$

The elements of the this group are

$$
\left\{1, a, \cdots, a^{2 n-1}, b, b a, \cdots, b a^{2 n-1}\right\} .
$$

One can prove that $Z\left(T_{4 n}\right)=\left\langle b^{2}\right\rangle$ and so $\left|Z\left(T_{4 n}\right)\right|=2$. This implies that

$$
\left|V\left(\Gamma\left(T_{4 n}\right)\right)\right|=\left|T_{4 n}\right|-\left|Z\left(T_{4 n}\right)\right|=4 n-2 .
$$


It is not difficult to see that, $\Gamma\left(T_{4 n}\right)$ is $(n+1)$-partite graph. On the other hand, $\Gamma$ has $2 n-2$ vertices of degree $2 n$ and $2 n$ vertices of degree $4 n-4$. This implies that the adjacency matrix of $\Gamma\left(T_{4 n}\right)$ is

$$
\left(\begin{array}{cccccc}
0_{2} & \cdots & 0_{2} & J_{2} & \cdots & J_{2} \\
\vdots & & & & & \\
0_{2} & \cdots & 0_{2} & J_{2} & \cdots & J_{2} \\
J_{2} & \cdots & J_{2} & 0_{2} & \cdots & J_{2} \\
\vdots & & & & & \\
J_{2} & \cdots & J_{2} & J_{2} & \cdots & 0_{2}
\end{array}\right)=\left(\begin{array}{cc}
0_{n-1} & J_{(n-1) \times n} \\
J_{n \times(n-1)} & (J-I)_{n}
\end{array}\right) \otimes J_{2}
$$

We recall that a finite group is called a $p$-group if and only if its order is a power of $p$, where $p$ is a prime integer. In [1]3], it is proved that there is no regular $N C$-graph of valency $p^{n}$, where $p$ is an odd prime number and $n$ is a positive integer. In general, we have the following result.

Theorem 2.4. [13] Let $G$ be a finite non-abelian group such that $\Gamma(G)$ is k-regular. Then $k$ is an even number greater than or equal with 4.

Theorem 2.5. [13] Let $G$ be a finite non-abelian group such that $\Gamma(G)$ is $2^{\text {s-regular, where }}$ $s \in \mathbb{N} \backslash\{1\}$. Then $G$ is a 2-group.

Proposition 2.6. [I] Let $G$ be a finite non-abelian group such that $\Gamma(G)$ is a regular graph. Then $G$ is nilpotent of class at most 3 and $G=P \times A$, where $A$ is an abelian group, $P$ is a p-group (p is a prime) and furthermore $\Gamma(P)$ is a regular graph.

Theorem 2.7. [14] Let $G$ be a non-abelian group and $p$ be a prime number. If $[G: Z(G)]=p^{2}$, then $\Gamma(G)$ is a regular graph.

Theorem 2.8. [6] Let $G$ be a finite non-abelian group. Then $|C e n t(G)|=4$ if and only if $G / Z(G) \cong \mathbb{Z}_{2} \times \mathbb{Z}_{2}$

Theorem 2.9. [6] Let $G$ be a finite non-abelian group and $p$ be a prime number. If $G / Z(G) \cong$ $\mathbb{Z}_{p} \times \mathbb{Z}_{p}$, then $|\operatorname{Cent}(G)|=p+2$.

Remark 2.10. Let $G \cong P \times \mathbb{Z}_{q}$ where $p, q$ are prime numbers and $P$ be a $p$-group. Hence, we have $G / Z(G) \cong P / Z(P)$. Thus, $P / Z(P) \cong \mathbb{Z}_{p} \times \mathbb{Z}_{p}$ if and only if $G / Z(G) \cong \mathbb{Z}_{p} \times \mathbb{Z}_{p}$.

Proposition 2.11. [13] Let $p$ be a prime number and $P$ be a non-abelian p-group. Then $\Gamma(P)$ is $k$-regular if and only if $\Gamma\left(P \times \mathbb{Z}_{q}\right)$ is kq-regular, where $q$ is a prime number.

In the following by $\bar{K}_{n}$ we mean the complement of the complete graph $K_{n}$. 
Alg. Struc. Appl. Vol. 4 No. 2 (2017) 27-38.

Corollary 2.12. [14] Let $p$ be a prime number and $P$ be a non-abelian p-group. If $G=P \times A$, where $A$ is an abelian group, then the graph $\Gamma(G)$ is lexicographic product of $\Gamma(P)$ around $\bar{K}_{|A|}$ i.e. $\Gamma(G) \cong \Gamma(P) o \bar{K}_{|A|}$.

Theorem 2.13. [14] Let $G$ be a finite non-abelian group and $p$ be a prime number. Then $G / Z(G) \cong \mathbb{Z}_{p} \times \mathbb{Z}_{p}$ if and only if $\Gamma(G)$ is a regular complete $(p+1)$-partite graph.

\section{Main Results}

Let $\Gamma$ be a graph with adjacency matrix $A$, the characteristic polynomial of $\Gamma$ is defined as $\chi_{\Gamma}(\lambda)=\operatorname{det}(\lambda I-A)$, where $I$ is the identity matrix. The roots of this polynomial are called the eigenvalues of $\Gamma$ and form the spectrum of this graph, see [4, ए0, П1, 15, 16, ए7]. It is a well-known fact that if $A$ is a real symmetric matrix, then all eigenvalues of $A$ are real. The graph $\Gamma$ is said to be integral if all its eigenvalues are integers, see [3, 15, 9, 10, 18].

Proposition 3.1. [22] A graph has exactly one positive eigenvalue if and only if the nonisolated vertices form a complete multipartite graph.

Lemma 3.2. [1]] Let $M$ be the following block matrix:

$$
M=\left(\begin{array}{cc}
0_{m \times m} & B_{m \times n} \\
B_{n \times m}^{T} & A_{n \times n}
\end{array}\right) .
$$

Then

$$
\chi_{M}(\lambda)=|\lambda I-M|=\lambda^{m-n}\left|\lambda^{2} I_{n}-\lambda A-B^{T} B\right| .
$$

Theorem 3.3. [8] Let $A$ and $B$ be square matrices of orders $m$ and $n$, respectively. If $\lambda_{1}, \cdots, \lambda_{m}$ are eigenvalues of $A$ and $\mu_{1}, \cdots, \mu_{n}$ are eigenvalues of $B$, then for $1 \leq i \leq m, 1 \leq$ $j \leq n$, the eigenvalues of $A \otimes B$ are $\lambda_{i} \mu_{j}$ and the eigenvalues of $A \otimes I_{m}+I_{n} \otimes B$ are $\lambda_{i}+\mu_{j}$.

The aim of this section is to study the spectral properties of $N C$-graphs. In [I] it is proved that the diameter of an $N C$-graph is two. On the other hand, in [10] it is proved that if $\Gamma$ is an integral $k$-regular graph on $n$ vertices with diameter $d$, then

$$
n \leq \frac{k(k-1)^{d}-2}{k-2} .
$$

By using these results, in [1]3] the authors has proposed a necessary condition for $\Gamma(G)$ to be an integral $k$-regular graph. Here, we give a sufficient condition for $\Gamma(G)$ to be integral.

Theorem 3.4. Let $G$ be a finite non-abelian group and $p$ be a prime number. If $G / Z(G) \cong$ $\mathbb{Z}_{p} \times \mathbb{Z}_{p}$, then $\Gamma(G)$ is an integral graph 
Proof. By Theorem [2.J3, $\Gamma(G)$ is a regular complete $(p+1)$-partite graph and so it is a strongly regular graph with parameters $(k, \lambda, \mu)$. Hence, the eigenvalues of $\Gamma(G)$ are as follows:

$$
\left\{\left[\frac{\lambda-\mu-\sqrt{(\lambda-\mu)^{2}+4(k-\mu)}}{2}\right]^{m_{1}},\left[\frac{\lambda-\mu+\sqrt{(\lambda-\mu)^{2}+4(k-\mu)}}{2}\right]^{m_{2}},[k]^{1}\right\} .
$$

If $n$ is the number of vertices of the graph, then the number of vertices of each part of this graph is $n /(p+1)$. Hence, we have

$$
k=\frac{p n}{p+1}, \lambda=\frac{(p-1) n}{p+1}, \mu=\frac{p n}{p+1} .
$$

Therefore the spectrum of $\Gamma(G)$ is

$$
\operatorname{Spec}(\Gamma(G))=\left\{\left[\frac{-n}{p+1}\right]^{m_{1}},[0]^{m_{2}},\left[\frac{p n}{p+1}\right]^{1}\right\} .
$$

On the other hand, $m_{1}+m_{2}+1=n$ and $\frac{p n}{p+1}+m_{1} \frac{-n}{p+1}=0$. Hence $m_{1}=p$ and $m_{2}=n-1-p$. Since $p+1$ divides $n$, the eigenvalues of this graph are integral. $\square$

Corollary 3.5. Let $G$ be a finite non-abelian group and $p$ be a prime number. If $G / Z(G) \cong$ $\mathbb{Z}_{p} \times \mathbb{Z}_{p}$, then $\Gamma(G)$ has only one positive eigenvalue. In particular, $p(p-1)|Z(G)|$ is the only positive eigenvalue of the regular graph $\Gamma(G)$.

Proof. According to Theorem [2.]3, $\Gamma(G)$ is a complete $(p+1)$-partite graph. Thus, by using Proposition [.], $\Gamma(G)$ has only one positive eigenvalue. By Theorem 3.4 the positive eigenvalue of $\Gamma(G)$ is

$$
\frac{p(|G|-|Z(G)|)}{p+1}=\frac{p\left(p^{2}-1\right)|Z(G)|}{p+1}=p(p-1)|Z(G)| .
$$

Theorem 3.6. Let $p$ be a prime number and $P$ be a p-group. If $G=P \times A$ where $A$ is an abelian group, then the spectrum of $\Gamma(G)$ is

$$
\left\{[0]^{(a-1)|V(\Gamma(P))|},\left[a \lambda_{1}\right]^{m_{1}}, \cdots,\left[a \lambda_{s}\right]^{m_{s}}\right\},
$$

where $\left\{\left[\lambda_{1}\right]^{m_{1}}, \cdots,\left[\lambda_{s}\right]^{m_{s}}\right\}$ is the spectrum of $\Gamma(P)$ and $|A|=a$.

Proof. By Corollary [.22, we have $\Gamma(G) \cong \Gamma(P) o \bar{K}_{|A|}$. Let $|A|=a$ and $B$ be the adjacency matrix of $\Gamma(P)$. Since the adjacency matrix of $\bar{K}_{|A|}$ is $(0)_{a \times a}$, the adjacency matrix of $\Gamma(G)$ is $B \otimes J_{a}$. Since the characteristic polynomial of $J_{a}$ is $\chi_{J_{a}}(\lambda)=\lambda^{a-1}(\lambda-a)$, by using Theorem [3.3, the spectrum of $\Gamma(G)$ is

$$
\operatorname{Spec}(\Gamma(G))=\left\{[0]^{(a-1)|V(\Gamma(P))|},\left[a \lambda_{1}\right]^{m_{1}}, \cdots,\left[a \lambda_{s}\right]^{m_{s}}\right\} .
$$


Lemma 3.7. Consider the block matrix

$$
A=\left(\begin{array}{cc}
0_{n-1} & J_{(n-1) \times n} \\
J_{n \times(n-1)} & (J-I)_{n}
\end{array}\right) .
$$

The characteristic polynomial of $A$ is

$$
\chi_{A}(\lambda)=\lambda^{n-2}(\lambda+1)^{n-1}\left(\lambda^{2}+(1-n) \lambda-n(n-1)\right) .
$$

Proof. By using Lemma 3.2, we have

$$
\chi_{A}(\lambda)=\lambda^{m-n}\left|\lambda^{2} I_{n}-\lambda A-B B^{T}\right|,
$$

where $m=n-1$ and $B=J_{n \times(n-1)}$. Hence,

$$
\chi_{A}(\lambda)=\lambda^{-1}\left|\left(\lambda^{2}+\lambda\right) I_{n}-(\lambda+n-1) J_{n}\right|=\lambda^{-1}(\lambda+n-1)^{n} \chi_{\frac{\lambda^{2}+\lambda}{\lambda+n-1}}\left(J_{n}\right) .
$$

Since $\chi_{J_{n}}(\lambda)=\lambda^{n-1}(\lambda-n)$, the proof is complete. $\square$

Theorem 3.8. The spectrum of $\Gamma\left(U_{6 n}\right)$ is

$$
\operatorname{Spec}\left(\Gamma\left(U_{6 n}\right)\right)=\left\{[-n]^{2},[n \pm n \sqrt{7}]^{1},[0]^{5 n-4}\right\} .
$$

Proof. In Example [2., it is shown that $\Gamma\left(U_{6 n}\right)$ is a 4-partite graph with the adjacency matrix $B \otimes J_{n}$. The eigenvalues of $J_{n}$ and $B$ are $\left\{[0]^{n-1},[n]^{1}\right\}$ and $\left\{[0]^{1},[1+\sqrt{7}]^{1},[1-\right.$ $\left.\sqrt{7}]^{1},[-1]^{2}\right\}$, respectively. Now Theorem $[3.3$ yeilds the proof. $\square$

Theorem 3.9. The spectrum of graph $\Gamma\left(T_{4 n}\right)$ is as follows:

$$
\operatorname{Spec}\left(\Gamma\left(T_{4 n}\right)\right)=\left\{[-2]^{n-1},[0]^{3 n-3},[(n-1) \pm \sqrt{(5 n-1)(n-1)}]^{1}\right\}
$$

Proof. In Example [2.3, it is shown that $\Gamma\left(T_{4 n}\right)$ is a $(n+1)$-partite graph with the following adjacency matrix

$$
\left(\begin{array}{cc}
0_{n-1} & J_{(n-1) \times n} \\
J_{n \times(n-1)} & (J-I)_{n}
\end{array}\right) \otimes J_{2} .
$$

The spectrum of the left hand matrix can be computed directly from Lemma $\mathbf{3 . 7}$ as follows:

$$
\left\{[-1]^{n-1},[0]^{n-2},\left[\frac{n-1}{2} \pm \frac{\sqrt{(5 n-1)(n-1)}}{2}\right]^{1}\right\} .
$$

Thus, by using Theorem B.3, the proof is complete. $\square$ 
Theorem 3.10. The spectrum of $N C$-graph $D_{2 n}$ is as follows:

if $n$ is odd:

$$
\operatorname{Spec}\left(\Gamma\left(D_{2 n}\right)\right)=\left\{[-1]^{n-1},[0]^{n-2},\left[\frac{n-1}{2} \pm \frac{\sqrt{(5 n-1)(n-1)}}{2}\right]^{1}\right\} .
$$

if $n$ is even:

$$
\operatorname{Spec}\left(\Gamma\left(D_{2 n}\right)\right)=\left\{[-2]^{\frac{n}{2}-1},[0]^{\frac{3 n}{2}-3},\left[\left(\frac{n}{2}-1\right) \pm \sqrt{\left(\frac{5 n}{2}-1\right)\left(\frac{n}{2}-1\right)}\right]^{1}\right\} .
$$

Proof. In finding the spectrum of $\Gamma\left(D_{2 n}\right)$, it is convenient to consider two separately cases: Case 1. $n$ is odd, the adjacency matrix of $\Gamma$ has the following form:

$$
\left(\begin{array}{cc}
0_{n-1} & J_{(n-1) \times n} \\
J_{n \times(n-1)} & (J-I)_{n}
\end{array}\right) .
$$

By using Lemma [3.7, the proof is complete.

Case 2. $n=2 m$ is even, in this case $\Gamma\left(D_{2 n}\right) \cong \Gamma\left(T_{4 m}\right)$ and according to Theorem [.. , the proof is complete. $\square$

Here, we determine the spectrum of $N C$-graph of group $V_{8 n}(n$ is odd) with the following presentation:

$$
V_{8 n}=\left\langle a, b: a^{2 n}=b^{4}=1, b^{-1} a b^{-1}=b a b=a^{-1}\right\rangle .
$$

Theorem 3.11. The spectrum of $\Gamma\left(V_{8 n}\right)$ is given by

$$
\left\{[-2]^{2 n-1},[0]^{6 n-3},\left[2 n-1 \pm \sqrt{20 n^{2}-12 n+1}\right]^{1}\right\}
$$

Proof. One can prove that $Z\left(V_{8 n}\right)=\left\langle b^{2}\right\rangle$ and so $\left|Z\left(V_{8 n}\right)\right|=2$. This implies that

$$
\left|V\left(\Gamma\left(V_{8 n}\right)\right)\right|=\left|V_{8 n}\right|-\left|Z\left(V_{8 n}\right)\right|=8 n-2 .
$$

Similar to the proof of Theorem $\overline{3.9}$ and Theorem $\mathbf{3 . 0}$, we can show that $\Gamma\left(V_{8 n}\right)$ is a $(2 n+1)$-partite graph with the following partitions:

$$
\begin{aligned}
V_{1} & =\left\{a, \cdots, a^{2 n-1}, a b^{2}, \cdots, a^{2 n-1} b^{2}\right\}, \\
V_{2} & =\left\{b, b^{3}\right\} \\
V_{3} & =\left\{a b, a b^{3}\right\} \\
\vdots & \\
V_{2 n+1} & =\left\{a^{2 n-1} b, a^{2 n-1} b^{3}\right\} .
\end{aligned}
$$


In other words, the vertices of $V_{1}$ have degree $4 n$ and the other vertices have degree $8 n-4$. This implies that its adjacency matrix is $A\left(\Gamma\left(V_{8 n}\right)\right)=C \otimes J_{2}$, where

$$
C=\left(\begin{array}{cc}
0_{2 n-1} & J_{(2 n-1) \times 2 n} \\
J_{2 n \times(2 n-1)} & (J-I)_{2 n}
\end{array}\right) .
$$

By using Lemma B.7, we have

$$
\chi_{C}(\lambda)=\lambda^{2 n-2}(\lambda+1)^{2 n-1}\left(\lambda^{2}+(1-2 n) \lambda-4 n^{2}+2 n\right) .
$$

By computing the roots of above polynomial, the spectrum of $C$ can be computed as follows:

$$
\left\{[-1]^{2 n-1},[0]^{2 n-2},\left[\left(2 n-1 \pm \sqrt{20 n^{2}-12 n+1}\right) / 2\right]^{1}\right\}
$$

Now, apply Theorem 3.3 to complete the proof. $\square$

In continuing, we determine the spectrum of $N C$-graph of group $S D_{8 n}$ with the following presentation:

$$
S D_{8 n}=\left\langle a, b: a^{4 n}=b^{2}=1, b a b=a^{2 n-1}\right\rangle .
$$

Theorem 3.12. The spectrum of $\Gamma\left(S D_{8 n}\right)$ is as follows:

if $n$ is even:

$$
\operatorname{Spec}\left(\Gamma\left(S D_{8 n}\right)\right)=\left\{[-2]^{2 n-1},[0]^{6 n-3},\left[2 n-1 \pm \sqrt{20 n^{2}-12 n+1}\right]^{1}\right\} .
$$

if $n$ is odd:

$$
\operatorname{Spec}\left(\Gamma\left(S D_{8 n}\right)\right)=\left\{[-4]^{n-1},[0]^{7 n-5},[2(n-1) \pm 2 \sqrt{(5 n-1)(n-1)}]^{1}\right\} .
$$

Proof. One can prove that if $n$ is even, then $Z\left(S D_{8 n}\right)=\left\langle a^{2 n}\right\rangle$ and so $\left|Z\left(S D_{8 n}\right)\right|=2$ and if $n$ is odd, then $Z\left(S D_{8 n}\right)=\left\langle a^{n}\right\rangle$. Thus, $\left|Z\left(S D_{8 n}\right)\right|=4$. This implies that if $n$ is even, then

$$
\left|V\left(\Gamma\left(S D_{8 n}\right)\right)\right|=\left|S D_{8 n}\right|-\left|Z\left(S D_{8 n}\right)\right|=8 n-2,
$$

and if $n$ is odd, then

$$
\left|V\left(\Gamma\left(S D_{8 n}\right)\right)\right|=\left|S D_{8 n}\right|-\left|Z\left(S D_{8 n}\right)\right|=8 n-4 .
$$

We can show that if $n$ is even then $\Gamma\left(S D_{8 n}\right)$ is a $(2 n+1)$-partite graph with partitions

$$
\begin{aligned}
V_{1} & =\left\{a, a^{2}, \cdots, a^{2 n-1}, a^{2 n+1}, \cdots, a^{4 n-1}\right\}, \\
V_{2} & =\left\{b, a^{2 n} b\right\} \\
V_{3} & =\left\{a b, a^{2 n+1} b\right\} \\
\vdots & \\
V_{2 n+1} & =\left\{a^{2 n-1} b, a^{4 n-1} b\right\}
\end{aligned}
$$


and if $n$ is odd then $\Gamma\left(S D_{8 n}\right)$ is a $(n+1)$-partite graph with partitions

$$
\begin{aligned}
V_{1} & =\left\{a, a^{2}, \cdots, a^{4 n-1}\right\} \backslash\left\{a^{n}, a^{2 n}, a^{3 n}\right\}, \\
V_{2} & =\left\{b, a^{n} b, a^{2 n} b, a^{3 n} b\right\}, \\
V_{3} & =\left\{a b, a^{n+1} b, a^{2 n+1} b, a^{3 n+1} b\right\}, \\
\vdots & \\
V_{n+1} & =\left\{a^{n-1} b, a^{2 n-1} b, a^{3 n-1} b, a^{4 n-1} b\right\} .
\end{aligned}
$$

In other words, if $n$ is even, then the vertices of $V_{1}$ have degree $4 n$ and the other vertices have degree $8 n-4$. This implies that its adjacency matrix is equal with $A\left(\Gamma\left(V_{8 n}\right)\right)$ and thus $\Gamma\left(S D_{8 n}\right)$ and $\Gamma\left(V_{8 n}\right)$, where $n$ is even, are co-spectral. If $n$ is odd, the vertices of $V_{1}$ have degree $4 n$ and the other vertices have degree $8 n-8$. This implies that its adjacency matrix is $A\left(\Gamma\left(S D_{8 n}\right)\right)=C \otimes J_{4}$, where

$$
C=\left(\begin{array}{cc}
0_{n-1} & J_{(n-1) \times n} \\
J_{n \times(n-1)} & (J-I)_{n}
\end{array}\right) .
$$

The spectrum of $C$ can be directly computed by Lemma 3.7 as follows:

$$
\left\{[-1]^{n-1},[0]^{n-2},\left[\frac{n-1}{2} \pm \frac{\sqrt{(5 n-1)(n-1)}}{2}\right]^{1}\right\} .
$$

Thus, by using Theorem 3.31 , the proof is complete.

Finally, we determine the spectrum of $N C$-graph of Frobenius group $F_{p, q}$ in which $p$ is prime and $q \mid p-1$. This group is a non-abelian group of order $p q$ with the following presentation:

$$
F_{p, q}=\left\langle a, b: a^{p}=b^{q}=1, b^{-1} a b=a^{u}\right\rangle
$$

where $u$ is an element of order $q$ in $\mathbb{Z}_{p}^{*}$.

Theorem 3.13. Let $\alpha=(q-1)(p-1)$. The spectrum of $\Gamma\left(F_{p, q}\right)$ is given by

$$
\left\{[-(q-1)]^{p-1},[0]^{p q-p-2},\left[\frac{\alpha \pm \sqrt{\alpha^{2}-4 p \alpha}}{2}\right]^{1}\right\} .
$$

Proof. It is not difficult to see that $Z\left(F_{p, q}\right)=1$ and therefore $\left|Z\left(F_{p, q}\right)\right|=1$. The elements of this group are

$$
\left\{1, a, a^{2}, \cdots, a^{p-1}\right\} \cup\left\{a^{m} b^{n} ; 0 \leq m \leq p-1,1 \leq n \leq q-1\right\} .
$$

Now we compute the centralizer of $a^{m} b^{n}$. First notice that

$$
\left[G: C_{G}\left(a^{m} b^{n}\right)\right]=\left|\left(a^{m} b^{n}\right)^{G}\right|=\left|\left(b^{n}\right)^{G}\right|=p .
$$


This implies that $\frac{|G|}{\mid C_{G}\left(a^{m} b^{n} \mid\right.}=p$ and so $\left|C_{G}\left(a^{m} b^{n}\right)\right|=q$ which yields $\left\langle a^{m} b^{n}\right\rangle \subseteq C_{G}\left(a^{m} b^{n}\right)$. On the other hand, $o\left(a^{m} b^{n}\right)=q$ and therefore $\left\langle a^{m} b^{n}\right\rangle=C_{G}\left(a^{m} b^{n}\right)$. So the graph $\Gamma\left(F_{p, q}\right)$ is a multi-partite graph, where one part is of order $p-1$ with the elements $\left\{1, a, a^{2}, \cdots, a^{p-1}\right\}$ and the other parts are of order $q-1$. Clearly, the number of parts of order $q-1$ is

$$
\frac{p q-(p-1)-1}{q-1}=\frac{p q-p}{q-1}=p .
$$

This implies that

$$
A\left(\Gamma\left(F_{p, q}\right)\right)=\left(\begin{array}{ccccc}
0_{p-1} & J_{(p-1) \times(q-1)} & J_{(p-1) \times(q-1)} & \cdots & J_{(p-1) \times(q-1)} \\
J_{(q-1) \times(p-1)} & 0_{(q-1)} & J_{(q-1)} & \cdots & J_{(q-1)} \\
\vdots & & & & \\
J_{(q-1) \times(p-1)} & J_{(q-1)} & \cdots & \cdots & 0_{(q-1)}
\end{array}\right) .
$$

This yields that

$$
\begin{aligned}
\operatorname{det}\left(x I-A\left(\Gamma\left(F_{p, q}\right)\right)\right) & =\left|\begin{array}{c|c}
x I_{(p-1)} & J_{1 \times p} \otimes\left(-J_{(p-1) \times(q-1)}\right) \\
\hline J_{p \times 1} \otimes\left(-J_{(q-1) \times(p-1)}\right) & I_{p} \otimes x I_{q-1}+(J-I)_{p} \otimes\left(-J_{(q-1)}\right)
\end{array}\right| \\
& =x^{p q-p-2}(x+(q-1))^{p-1}\left(x-x_{1}\right)\left(x-x_{2}\right) .
\end{aligned}
$$

where

$$
x_{1}=\frac{\alpha+\sqrt{\alpha^{2}-4 p \alpha}}{2} \text { and } x_{2}=\frac{\alpha-\sqrt{\alpha^{2}-4 p \alpha}}{2} .
$$

This completes the proof.

\section{REFERENCES}

[1] A. Abdollahi, S. Akbari, H. R. Maimani, Non-commuting graph of a group, J. Algebra 298 (2006) 468-492.

[2] A. Abdollahi, S.M.J. Amiri, A.M. Hassanabadi, Groups with specific number of centralizers, Houston J. Math. 33(1) (2007) 43-57.

[3] O. Ahmadi, N. Alon, L. F. Blake, I. E. Shparlinski, Graphs with integral spectrum, Linear Alg. Appl. 430 (2009) 547-552.

[4] S. J. Baishya, On finite groups with specific number of centralizers, Int. Electronic J. Algebra 13 (2013) 53-62.

[5] K. Balinska, D. Cvetković, Z. Rodosavljević, S. Simić, D. A. Stevanović, Survey on integral graphs, Univ. Beograd, Publ. Elektrotehn. Fak. Ser. Mat. 13 (2003) 42-65.

[6] S. M. Belcastro, G. J. Sherma, Counting centralizers in finite groups, Math. Magazine 67(5) (1994) 366-374.

[7] N. L. Biggs, Algebraic Graph Theory, Cambridge University Press, 1974.

[8] R. A. Brualdi, D. Cvetkovic, A Combinatorial Approach to Matrix Theory and Its Applications, Chapman and Hall/CRC; Second edition, 2008. 
[9] F. C. Bussemaker, D. Cvetković, There are exactly 13 connected, cubic, integral graphs, Univ. Beograd, Publ. Elektrotehn. Fak. Ser. Mat. Fiz. 544-576 (1976) 43-48.

[10] D. Cvetković, Cubic integral graphs, Univ. Beograd, Publ. Elektrotehn. Fak. Ser. Mat. Fiz. 498-541 (1975) 107-113.

[11] D. Cvetković, P. Rowlinson, S. Simić, An introduction to the theory of graph spectra, London Mathematical Society, London, 2010.

[12] M. R. Darafsheh, Groups with the same non-commuting graph, Discrete Appl. Math. 157 (2009) $833-837$.

[13] M. Ghorbani, Z. Gharavi-Alkhansari, A note on integral non-commuting graphs, Filomat 313 (2017) 663669.

[14] M. Ghorbani, Z. Gharavi-Alkhansari, Some properties of non-commuting graphs, submited.

[15] M. Ghorbani, F. Nowroozi-Larki, On the spectrum of finite Cayley graphs, Journal of Discrete Mathematical Sciences and Cryptography 21 (2018) 83-112.

[16] M. Ghorbani, F. Nowroozi-Larki, On the Spectrum of Cayley Graphs Related to the Finite Groups, Filomat 31 (2017) 6419-6429.

[17] M. Ghorbani, F. Nowroozi-Larki, On the spectrum of Cayley graphs, Sib. Elektron. Mat. Izv. 13 (2016) 1283-1289.

[18] F. Harary, A. J. Schwenk, Which graphs have integral spectra?, in: R. Bari, F. Harary (Eds.), Graphs and Combinatorics, Lecture Notes in Mathematics, 406, Springer, Berlin (1974) 45-51.

[19] A. R. Moghaddamfar, W. J. Shi, W. Zhou, A. R. Zokayi, On the non-commuting graph associated with a finite group, Siberian Math. J. 46 (2005) 325-332.

[20] G. L. Morgan, C. W. Parker, The diameter of the commuting graph of a finite group with trivial centre, J. Algebra 393 (2013) 41-59.

[21] B. H. Neumann, A problem of Paul Erdős on groups, J. Austral. Math. Soc. Ser. A 21 (1976) 467-472.

[22] J. H. Smith, Some properties of the spectrum of a graph, Combinatorial Structures and their Applications, Gordon and Breach, New York (1970) 403-406.

\section{Modjtaba Ghorbani}

Department of Mathematics, Faculty of Science, Shahid Rajaee

Teacher Training University, Tehran, 16785-136, I. R. Iran

mghorbani@sru.ac.ir

\section{Zahra Gharavi}

Department of Mathematics, Faculty of Science, Shahid Rajaee

Teacher Training University, Tehran, 16785-136, I. R. Iran

z.gh.alk5@gmail.com

\section{Ali Zaeem-Bashi}

Department of Mathematics, Faculty of Science, Shahid Rajaee

Teacher Training University, Tehran, 16785-136, I. R. Iran

zaeembashiali@yahoo.com 